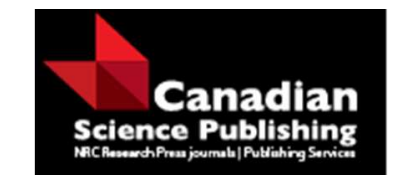

Canadian Journal of Physics

Revue canadienne de physique

\title{
Analytical study of holographic superconductors with exponential nonlinear electrodynamics
}

\begin{tabular}{|r|l|}
\hline Journal: & Canadian Journal of Physics \\
\hline Manuscript ID & cjp-2016-0526 \\
\hline Manuscript Type: & Article \\
\hline Date Submitted by the Author: & 27-Jul-2016 \\
\hline Complete List of Authors: & $\begin{array}{l}\text { Sheykhi, Ahmad; Shahid Bahonar Univ, Physics } \\
\text { Shaker, Fatemeh; Shiraz University }\end{array}$ \\
\hline Keyword: & $\begin{array}{l}\text { holographic superconductor, critical temperature, exponential nonlinear, } \\
\text { electrodynamics, charge density }\end{array}$ \\
\hline &
\end{tabular}

SCHOLARONE ${ }^{\text {IM }}$

Manuscripts 


\title{
Analytical study of holographic superconductors with exponential nonlinear electrodynamics
}

\author{
A. Sheykhi ${ }^{1,2 *}$ and F. Shaker ${ }^{1}$ \\ 1 Physics Department and Biruni Observatory, College of Sciences, Shiraz University, Shiraz 71454, Iran \\ ${ }^{2}$ Research Institute for Astronomy and Astrophysics of Maragha (RIAAM), P.O. Box 55134-441, Maragha, Iran
}

\begin{abstract}
Based on the Sturm-Liouville (SL) eigenvalue problem, we analytically study several properties of holographic $s$-wave superconductors with Exponential Nonlinear (EN) electrodynamics in the background of Schwarzschild Anti-de Sitter (AdS) black holes. We assume the probe limit in which the scalar and gauge fields do not back react on the background metric. We show that for this system, one can still obtain an analytical relation between the critical temperature and the charge density. Interestingly enough, we find that EN electrodynamics decreases the critical temperature, $T_{c}$, of the holographic superconductors compared to the linear Maxwell field. This implies that the nonlinear electrodynamics make the condensation harder. The analytical results obtained in this paper are in good agreement with the existing numerical results. We also compute the critical exponent near the critical temperature and find out that it is still $1 / 2$ which seems to be an universal value in mean field theory.
\end{abstract}

Keywords: holographic superconductor; nonlinear electrodynamics; critical temperature

\section{INTRODUCTION}

Nowadays, the correspondence between an anti-de Sitter spacetime and a conformal field theory living on its boundary (AdS/CFT) is the well-known holographic picture of the world. This correspondence provides a method for relating a strongly interacting field theory to a dual classical gravity [1-3]. It was shown that by employing the dictionary of AdS/CFT duality, one can relate the observed properties in the AdS bulk to their corresponding quantities in CFT on the boundary. An interesting application of this duality was recently proposed by Hartnoll, et. al., $[4,5]$ who showed that some properties of strongly coupled superconductors can be potentially described by a classical general relativity living in one higher dimension. The motivation for this study is to shed some light on the problem of understanding the mechanism of the high temperature superconductors in condensed matter physics. Following $[4,5]$, a lot of works have been appeared which attempt to investigate the holographic superconductors from different perspective [6-19]. Focusing on the simplest form by working in the probe approximation, in which the scalar and gauge fields backreaction effects on the gravity sector can be ignored and fixing the metric as static, the authors of [20] employed the SL variational method as an analytic approach for calculating the properties of the holographic superconductors. They discussed analytic approximations to the solutions of the nonlinear field equations both near the critical temperature $T_{c}$ and in the low temperature limit $T \rightarrow 0$. The critical temperature and critical exponent and other quantities for different modes of superconductors including $s$-wave, $p$-wave and $d$-wave, were also investigated in the probe limit [21-25].

In description of the holographic dual models, some attempts have been done to generalize the linear Maxwell electrodynamics to the nonlinear electrodynamics. The motivation is to examine the effects of the higher order corrections to the Maxwell field on the properties and critical temperature of the holographic superconductors. In this regards, the authors of $[26,27]$ analytically studied the holographic superconductors in the presence of the Born-Infeld (BI) nonlinear electrodynamics by using the SL eigenvalue problem. They observed that the higher BI corrections make it harder for the condensation to form but do not affect the critical phenomena of the system. Also, the authors of [28] numerically studied the non-equilibrium condensation process in a holographic superconductor when the gauge field is in form of the BI and Logarithmic one.

Among the nonlinear corrections to the linear Maxwell field, it is of interest to consider the EN electrodynamics $[29,30]$. Numerical studies on the properties of the holographic superconductors in the presence of BI nonlinear electrodynamics [31] as well as Logarithmic and EN electrodynamics have been carried out [31, 32]. In particular, it was shown that EN electrodynamics correction makes the condensation harder [32]. When the gauge field is the BI one, based on the SL eigenvalue problem, several properties of holographic $s$-wave superconductors in the background of a Schwarzschild-AdS spacetime have been investigated in the prob limit $[33,35]$ and away from the probe limit $[36,37]$. As far as we know the analytical study on the properties of holographic superconductors 
with EN electrodynamics remains to be done. In this paper, we would like to extend the analytical study on the holographic $s$-wave superconductors by taking the gauge field in the form of EN electrodynamics and using SL analytical techniques. For economic reasons, we shall concentrate on the probe limit by neglecting backreaction of the matter fields on the spacetime metric. We shall compute the critical temperature and critical exponent for this kind of holographic superconductor by using the perturbation method. Then, we will compare our results with the existing numerical results for holographic superconductors with EN electrodynamics [32] as well as the analytical results derived in [33] for the holographic superconductors with BI nonlinear electrodynamics. Other studies on the holographic superconductors with nonlinear gauge field sources were carried out in [34].

The plan of this paper is as follows. In section II, we review the field equations and study the $(2+1)$-holographic superconductor in the framework of planar AdS black holes when the gauge field is in the form of EN electrodynamics. In section III, we calculate the critical temperature in terms of the charge density. In section IV, we continue the calculation for the condensations of the scalar operators and find an analytical expression for it. We show that the analytic study is comparable with the numerical computation. Finally, section V contains our concluding remarks.

\section{BASIC EQUATIONS}

We study the formation of scalar hair on the background of AdS black hole in 4-dimensions. In order to construct a holographic $s$-wave superconductor in the probe limit, we consider the background of the 4-dimensional planar Schwarzschild-AdS black hole

$$
d s^{2}=-f(r) d t^{2}+\frac{1}{f(r)} d r^{2}+r^{2}\left(d x^{2}+d y^{2}\right)
$$

where the metric function is

$$
f(r)=r^{2}-\frac{r_{+}^{3}}{r}
$$

Here $r_{+}$is the horizon radius of the black hole and we have taken the AdS radius equal unity, i.e. $l=1$. The Hawking temperature of the Schwarzschild-AdS black hole can be expressed as

$$
T=\frac{3 r_{+}}{4 \pi} .
$$

The Lagrangian density containing a nonlinear gauge field and a charged complex scalar field reads

$$
\mathcal{L}=\mathcal{L}(F)-|\nabla \psi-i q A \psi|^{2}-m^{2}|\psi|^{2}
$$

where $A$ and $\psi$ are, respectively, the gauge and scalar field with charge $q, \mathcal{L}(F)$ denotes the Lagrangian density of the EN electrodynamics and we take it as [29]

$$
\mathcal{L}(\mathcal{F})=\frac{1}{4 b}\left(e^{-b \mathcal{F}}-1\right)
$$

where $b$ is the nonlinear parameter, $\mathcal{F}=F_{\mu \nu} F^{\mu \nu}$, and $F^{\mu \nu}$ is the electromagnetic field tensor. When $b \rightarrow 0$, the nonlinear electrodynamic Lagrangian reduces to the linear Maxwell Lagrangian, $\mathcal{L}(\mathcal{F})=-\mathcal{F} / 4$. We further assume the gauge and scalar fields have the following form [4]

$$
A_{\mu}=(\phi(r), 0,0,0), \quad \psi=\psi(r)
$$

By varying the Lagrangian (4) with respect to the gauge field $\phi$ and scalar field $\psi$, we obtain the field equations,

$$
\begin{aligned}
& \phi^{\prime \prime}(r)\left(1+4 b \phi^{\prime 2}(r)\right)+\frac{2}{r} \phi^{\prime}(r)-\frac{2 \phi(r) \psi^{2}(r)}{f(r)} e^{-2 b \phi^{\prime 2}(r)}=0, \\
& \psi^{\prime \prime}(r)+\left(\frac{f^{\prime}(r)}{f(r)}+\frac{2}{r}\right) \psi^{\prime}(r)+\left(\frac{\phi^{2}(r)}{f^{2}(r)}-\frac{m^{2}}{f(r)}\right) \psi(r)=0 .
\end{aligned}
$$

It is clear that the nonlinear gauge field do not affect the equation of $\psi$, however, the equation for $\phi$ will be modified as given in (7). When $b \rightarrow 0$, Eq. (7) restores the corresponding equation of the standard holographic superconductor 
in Maxwell theory [4]. In order to do an analytic calculations, we define a new variable $z=r_{+} / r$. In terms of this new variable, Eqs. (7) and (8) become

$$
\begin{gathered}
\phi^{\prime \prime}(z)\left(1+4 b \frac{z^{4}}{r_{+}^{2}} \phi^{\prime 2}\right)+\frac{8 b z^{3}}{r_{+}^{2}} \phi^{\prime 3}(z)-\frac{2 \psi^{2}(z)}{z^{2}\left(1-z^{3}\right)} e^{-\frac{2 b z^{4}}{r_{+}^{2}} \phi^{\prime 2}(z)} \phi(z)=0, \\
\psi^{\prime \prime}(z)-\frac{2+z^{3}}{z\left(1-z^{3}\right)} \psi^{\prime}(z)+\left[\frac{\phi^{2}(z)}{r_{+}^{2}\left(1-z^{3}\right)^{2}}-\frac{m^{2}}{z^{2}\left(1-z^{3}\right)}\right] \psi(z)=0,
\end{gathered}
$$

where we have also used

$$
f(z)=r_{+}^{2}\left(\frac{1}{z^{2}}-z\right) .
$$

In order to avoid the divergence near the horizon, we assume the boundary conditions $\phi(z=1)=0$ and $\psi(z=1)=$ $\frac{3}{2} \psi^{\prime}(z=1)$. Also, on the boundary regime where $z \rightarrow 0$, the asymptotic solutions for Eqs. (9) and (10) are

$$
\phi \approx \mu-\frac{\rho}{r_{+}} z
$$

and

$$
\psi(z) \approx \frac{\psi_{-}}{r_{+}^{\Delta_{-}}} z^{\Delta_{-}}+\frac{\psi_{+}}{r_{+}^{\Delta_{+}}} z^{\Delta_{+}}
$$

where

$$
\Delta_{ \pm}=\frac{3 \pm \sqrt{9+4 m^{2}}}{2}
$$

and $\mu$ and $\rho$ are interpreted as the chemical potential and charge density of the dual field theory [4, 5]. Also $\psi_{ \pm}=\left\langle\mathcal{O}_{ \pm}>\right.$specifies the vacuum expectation value of the dual operator $\mathcal{O}_{ \pm}$on the boundary. According to the AdS/CFT correspondence, either $\psi_{+}$or $\psi_{-}$will act as a condensation operator while the other will act as a source $[4,5]$. In the present work, we choose $\psi_{-}=\left\langle\mathcal{O}_{-}\right\rangle$and $\psi_{+}$as its source. Since we would like the condensation to take place in the absence of any source, we set $\psi_{+}=0$. Considering the fact that $m^{2}$ must satisfy the BreitenlohnerFreedman (BF) bound [38], we set $m^{2}=-2[5]$.

\section{ANALYTICAL INVESTIGATION OF THE HOLOGRAPHIC SUPERCONDUCTOR}

In this section, we would like to analytically study the relation between the critical temperature and charge density for the holographic superconductor in the presence of EN electrodynamics. We shall employ the SL variational method. At the critical temperature, $T=T_{c}$, we have $\psi=0$ and hence Eq. (9) reduces to

$$
\phi^{\prime \prime}(z)\left(1+4 b \frac{z^{4}}{r_{+c}^{2}} \phi^{\prime 2}\right)+\frac{8 b z^{3}}{r_{+c}^{2}} \phi^{\prime 3}(z)=0 .
$$

The above equation has the following solution,

$$
\phi(z)=\int_{1}^{z} d z \frac{r_{+c}}{2 z^{2} \sqrt{b}} \sqrt{L_{W}\left(\frac{4 b z^{4} \beta^{2}}{r_{+c}^{2}}\right)},
$$

where $\beta$ is a constant of integration and $L_{W}(x)=\operatorname{Lambert} W(x)$ is the Lambert function which satisfies [39]

$$
L_{W}(x) e^{L_{W}(x)}=x,
$$

and has the following series expansion

$$
L_{W}(x)=x-x^{2}+\frac{3}{2} x^{3}-\frac{8}{3} x^{4}+\ldots
$$


Clearly, series (18) converges for $|x|<1$. Expanding Eq. (16) and keeping terms up to $\mathcal{O}(b)$, we find

$$
\phi(z)=-\beta(1-z)+\frac{2 \beta^{3} b}{5 r_{+c}^{2}}\left(1-z^{5}\right)+\mathcal{O}\left(b^{2}\right)
$$

Equating $\phi^{\prime}(z=0)$ from Eq. (19) and Eq. (12), we find $\beta=-\rho / r_{+c}$, where at $T=T_{c}$ we have set $r_{+}=r_{+c}$. Substituting $\beta$ into Eq. (19), we arrive at

$$
\begin{aligned}
\phi(z) & =\frac{\rho}{r_{+c}}(1-z)-\frac{2 \rho^{3} b}{5 r_{+c}^{5}}\left(1-z^{5}\right) \\
& =\frac{\rho}{r_{+c}}(1-z)\left\{1-\frac{2 \rho^{2}}{5 r_{+c}^{4}} b\left(1+z+z^{2}+z^{3}+z^{4}\right)\right\} \\
& =\lambda r_{+c}(1-z)\left\{1-\frac{2}{5} b \lambda^{2}\left(1+z+z^{2}+z^{3}+z^{4}\right)\right\},
\end{aligned}
$$

with $b \lambda^{2}<1$, and in the last step we have defined

$$
\lambda=\frac{\rho}{r_{+c}^{2}} .
$$

It should be noted that we have investigated the effects of the nonlinear corrections up to the leading order in the nonlinear parameter $b$. In the next step, we start from the field equation for the scalar field $\psi$ by writing it near the critical temperatures $T \approx T_{c}$. Thus Eq. (10) can be rewritten as

$$
\begin{aligned}
& \psi^{\prime \prime}(z)-\frac{2+z^{3}}{z\left(1-z^{3}\right)} \psi^{\prime}(z)-\frac{m^{2}}{z^{2}\left(1-z^{3}\right)} \psi(z) \\
& +\frac{\lambda^{2}}{r_{+}^{2}\left(1+z+z^{2}\right)^{2}}\left(1-\frac{2 b \lambda^{2}}{5} \zeta(z)\right) \psi(z)=0,
\end{aligned}
$$

where $\zeta(z)=1+z+z^{2}+z^{3}+z^{4}$, and we have used Eq. (20) as well. In order to match the behavior at the boundary one defines $\psi$ in the following form

$$
\psi(z)=\frac{<\mathcal{O}_{-}>}{\sqrt{2} r_{+}} z F(z)
$$

where we introduce a trial function

$$
F(z)=1-\alpha z^{2}
$$

which satisfies the boundary conditions $F(0)=1$ and $F^{\prime}(0)=0$ near the boundary $z=0$ [12]. Inserting Eq. (23) into Eq. (22), one can show that the trial function satisfies the following second-order SL self adjoint differential equation

$$
\left(1-z^{3}\right) F^{\prime \prime}(z)-3 z^{2} F^{\prime}(z)-z F(z)+\frac{\lambda^{2}(1-z)}{\left(1+z+z^{2}\right)^{2}}\left(1-\frac{4 b \lambda^{2}}{5} \zeta(z)\right) F(z)=0 .
$$

The above equation is solved subject to the boundary condition $F^{\prime}(0)=0$. The expression for estimating the minimum eigenvalue of $\lambda^{2}$ is provided by [33]

$$
\lambda^{2}=\frac{\int_{0}^{1} d z\left[p(z)\left[F^{\prime}(z)\right]^{2}+z F^{2}(z)\right]}{\int_{0}^{1} d z q(z)[F(z)]^{2}}
$$

where

$$
\begin{aligned}
& p(z)=1-z^{3} \\
& q(z)=\frac{1-z}{1+z+z^{2}}\left(1-\left.\frac{4}{5} b \lambda^{2}\right|_{b=0} \zeta(z)\right) .
\end{aligned}
$$


Here we have performed a perturbative expansion $b \lambda^{2}$ and retain only the terms that are linear in $b$ such that

$$
b \lambda^{2}=b\left(\left.\lambda^{2}\right|_{b=0}\right)+\mathcal{O}\left(b^{2}\right)
$$

where $\left.\lambda^{2}\right|_{b=0}$ is the value of $\lambda^{2}$ for $b=0$. In fact, we have only retain the terms that are linear in nonlinear parameter $b$. Having Eqs. (3) and (21) in mind, the critical temperature $T_{c}$ in terms of the charge density $\rho$ can be obtained as

$$
T_{c}=\frac{3}{4 \pi} \sqrt{\frac{\rho}{\lambda}}
$$

Our strategy for calculating the critical temperature for condensation is to minimize the function (26) with respect to the coefficient $\alpha$ and then substituting the minimum value of $\lambda$ in Eq.(30) we find the maximum value of $T_{c}$. For $b=0$, one gets

$$
\lambda^{2}=\frac{10 \alpha^{2}-6 \alpha+6}{(2 \pi+4 \pi \alpha) \sqrt{3}+\left(12 \alpha+12 \alpha^{2}-6\right) \ln (3)-36 \alpha-13 \alpha^{2}},
$$

whose minimum is $\lambda_{\min }^{2}=1.2683$ at $\alpha=0.2389$. For this value of $\lambda$, the critical temperature reads $T_{c}=0.2250 \sqrt{\rho}$. For other values of the nonlinear parameter $b$, we have summarized our results in table 1.

\begin{tabular}{|c|c|c|c|}
\hline$b$ & $\alpha$ & $\lambda_{\min }^{2}$ & $T_{c}$ \\
\hline 0 & 0.2389 & 1.2683 & $0.2250 \sqrt{\rho}$ \\
\hline 0.1 & 0.2449 & 1.4892 & $0.2161 \sqrt{\rho}$ \\
\hline 0.2 & 0.2533 & 1.8028 & $0.2060 \sqrt{\rho}$ \\
\hline 0.3 & 0.2660 & 2.2825 & $0.1942 \sqrt{\rho}$ \\
\hline
\end{tabular}

Table 1: The values of the eigenvalue $\lambda_{\text {min }}^{2}$, parameter $\alpha$ and the critical temperature $T_{c}$ for several values of the nonlinear parameter $b$.

We have compared in table 2 the numerical and analytical results for the critical temperature of holographic superconductors in the presence of two kind of nonlinear gauge field, namely BI nonlinear electrodynamics [32, 36] and EN electrodynamics [32]. In this table, the analytical results for BI case are taken from [36] (which also coincide with those obtained in [33]) and the numerical result for both BI and EN are invoked from [32]. We observe that for both nonlinear electromagnetic models, the critical temperature $T_{c}$ decreases with increasing the nonlinear parameter $b$. Also for the fixed values of the nonlinear parameter $b$, the critical temperature $T_{c}$ for BI form is larger compared with the EN form. This implies that in case of the EN electrodynamics, the condensation is more difficult to create with respect to the BI nonlinear electrodynamics. Our analytical results are also consistent with the numerical results obtained in Ref. [32]. Indeed, from table 2, we see that the values obtained through both analytical and numerical results are in the same order and the difference is very small. This difference may be due to the perturbative technique, where we have only considered terms up to the first order in the nonlinear coupling parameter $b$. But in both of these approaches the critical temperature, $T_{c}$, decreases with increasing the values of the nonlinear parameter $b$.

\begin{tabular}{|c|c|c|c|c|}
\hline$b$ & $\left.T_{c}^{\mathrm{EN}}\right|_{\text {Analytical }}$ & $\left.T_{c}^{\mathrm{EN}}\right|_{\text {Numerical }}$ & $\left.T_{c}^{\mathrm{BI}}\right|_{\text {Analytical }}$ & $\left.T_{c}^{\mathrm{BI}}\right|_{\text {Numerical }}$ \\
\hline 0 & $0.2250 \sqrt{\rho}$ & $0.2255 \sqrt{\rho}$ & $0.2250 \sqrt{\rho}$ & $0.2255 \sqrt{\rho}$ \\
\hline 0.1 & $0.2161 \sqrt{\rho}$ & $0.2247 \sqrt{\rho}$ & $0.2228 \sqrt{\rho}$ & $0.2253 \sqrt{\rho}$ \\
\hline 0.2 & $0.2060 \sqrt{\rho}$ & $0.2225 \sqrt{\rho}$ & $0.2206 \sqrt{\rho}$ & $0.2247 \sqrt{\rho}$ \\
\hline 0.3 & $0.1942 \sqrt{\rho}$ & $0.2217 \sqrt{\rho}$ & $0.2184 \sqrt{\rho}$ & $0.2237 \sqrt{\rho}$ \\
\hline
\end{tabular}

Table 2: The critical temperature $T_{c}$ for different values of the nonlinear parameter $b$ for EN and BI electrodynamics obtained by the analytical SL and numerical methods.

\section{CRITICAL EXPONENT AND CONDENSATION VALUES}

In this section, we would like to calculate the condensation value in the boundary field theory. We start from Eq. (9) by writing it near the critical point. Substituting Eq. (23) into Eq. (9), we arrive at

$$
\phi^{\prime \prime}(z)\left(1+4 b \frac{z^{4}}{r_{+}^{2}} \phi^{\prime 2}\right)+\frac{8 b z^{3}}{r_{+}^{2}} \phi^{\prime 3}(z)=\frac{\left\langle\mathcal{O}_{-}\right\rangle^{2}}{r_{+}^{2}} \frac{F^{2}(z)}{1-z^{3}}\left[1-\frac{2 b z^{4}}{r_{+}^{2}} \phi^{\prime 2}(z)\right] \phi(z)+\mathcal{O}\left(b^{2}\right) .
$$


where we have used

$$
\exp \left(-\frac{2 b z^{4}}{r_{+}^{2}} \phi^{\prime 2}(z)\right)=1-\frac{2 b z^{4}}{r_{+}^{2}} \phi^{\prime 2}(z)+\mathcal{O}\left(b^{2}\right)
$$

Then, we expand $\phi(z)$ perturbatively in the small parameter $\langle\mathcal{O}\rangle^{2} / r_{+}^{2}$ as follows:

$$
\frac{\phi(z)}{r_{+}}=\lambda(1-z)\left\{1-\frac{2 b \lambda^{2}}{5} \zeta(z)\right\}+\frac{\left\langle\mathcal{O}_{-}\right\rangle^{2}}{r_{+}^{2}} \chi(z)+\ldots
$$

Substituting Eq. (34) into (32) in first order with respect to the $\langle\mathcal{O}\rangle^{2} / r_{+}^{2}$, we obtain the following differential equation

$$
\chi^{\prime \prime}(z)\left[1+4 b \lambda^{2} z^{4}\right]+24 b \lambda^{2} z^{3} \chi^{\prime}(z)=\frac{\lambda F^{2}(z)}{1+z+z^{2}}\left\{1-\frac{2 b \lambda^{2}}{5}\left(\zeta(z)+5 z^{4}\right)\right\},
$$

where $\chi(z)$ satisfies the boundary condition

$$
\chi(1)=\chi^{\prime}(1)=0 .
$$

Multiplying both side of (35) by factor $\left(1+4 b \lambda^{2} z^{4}\right)^{1 / 2}$, we can write it as

$$
\frac{d}{d z}\left[\left(1+4 b \lambda^{2} z^{4}\right)^{3 / 2} \chi^{\prime}(z)\right]=\left(1+4 b \lambda^{2} z^{4}\right)^{1 / 2} \frac{\lambda F^{2}(z)}{1+z+z^{2}}\left\{1-\frac{2 b \lambda^{2}}{5}\left(\zeta(z)+5 z^{4}\right)\right\} .
$$

Using the boundary condition (36) and integrating Eq. (37) in the interval $[0,1]$, we finally obtain

$$
\chi^{\prime}(0)=-\lambda \mathcal{A},
$$

where

$$
\mathcal{A}=\int_{0}^{1} d z\left(1+4 b \lambda^{2} z^{4}\right)^{1 / 2} \frac{F^{2}(z)}{1+z+z^{2}}\left\{1-\frac{2 b \lambda^{2}}{5}\left(\zeta(z)+5 z^{4}\right)\right\} .
$$

Combining Eqs. (12) and (34), we may write

$$
\begin{aligned}
\frac{\mu}{r_{+}}-\frac{\rho}{r_{+}^{2}} z & =\lambda(1-z)\left\{1-\frac{2 b \lambda^{2}}{5} \xi(z)\right\}+\frac{\left\langle\mathcal{O}_{-}\right\rangle^{2}}{r_{+}^{2}} \chi(z) \\
& =\lambda(1-z)\left\{1-\frac{2 b \lambda^{2}}{5} \xi(z)\right\}+\frac{\left\langle\mathcal{O}_{-}\right\rangle^{2}}{r_{+}^{2}}\left(\chi(0)+z \chi^{\prime}(0)+\ldots\right),
\end{aligned}
$$

where in the last step we have expanded $\chi(z)$ around $z=0$. Now, if we equate the coefficients of $z$ on both sides of Eq. (40), we get

$$
\frac{\rho}{r_{+}^{2}}=\lambda-\frac{\left\langle\mathcal{O}_{-}\right\rangle^{2}}{r_{+}^{2}} \chi^{\prime}(0)=\lambda\left(1+\frac{\left\langle\mathcal{O}_{-}\right\rangle^{2}}{r_{+}^{2}} \mathcal{A}\right)
$$

Substituting $\lambda=\rho / r_{+c}^{2}$ into Eq. (41), and using the definition for the temperature given in (3), we finally get the expression for the order parameter $\left\langle\mathcal{O}_{-}\right\rangle$near the critical temperature $T_{c}$ as

$$
\left\langle\mathcal{O}_{-}\right\rangle=\gamma T_{c} \sqrt{1-\frac{T}{T_{c}}},
$$

where

$$
\gamma=\frac{4 \pi \sqrt{2}}{\sqrt{3 \mathcal{A}}}
$$

From Eq. (42) we find that the critical exponent of the system always takes the mean field value $1 / 2$. This result is independent of the nonlinear parameter $b$. Also, it is clear that $\left\langle\mathcal{O}_{-}\right\rangle$is zero at $T=T_{c}$ and condensation occurs for 
$T<T_{c}$. In table 3, we present the condensation values $\gamma$ obtained for a $(2+1)$-dimensional holographic superconductor, by the analytical SL method for both BI and EN electrodynamics and for different values of the nonlinear parameter $b$ Again, the analytical results for BI case are taken from [33]. In both cases, we observe that the condensation value $\gamma$ increases with increasing the nonlinear parameter $b$. This indicates that the higher nonlinear electrodynamics corrections to the gauge field make the condensation to be formed harder. On the other hand, comparing these two types nonlinear electrodynamics, we find that the condensation operator for the EN is larger than that of BI which means that the EN electrodynamics makes the condensation harder with respect to the BI nonlinear electrodynamics.

\begin{tabular}{|c|c|c|}
\hline$b$ & $\gamma^{\mathrm{EN}}$ & $\gamma^{\mathrm{BI}}$ \\
\hline 0 & 8.07 & 8.07 \\
\hline 0.1 & 8.53 & 8.19 \\
\hline 0.2 & 9.16 & 8.33 \\
\hline 0.3 & 10.03 & 8.54 \\
\hline
\end{tabular}

Table 3: A comparison of the analytical results for the expectation value of the condensation operator, $\gamma$, for EN and BI electrodynamics.

\section{CONCLUDING REMARKS}

In the present paper, we analytically studied the properties of the holographic $s$-wave superconductors in the presence of exponential correction to the Maxwell electrodynamics in the background of Schwarzschild AdS black holes. Adopting the probe limit, where the scalar and gauge field do not back react on the metric background, we found the nonlinear coupling parameter influences the critical temperature and the condensation operator near the critical point. We performed our calculations up to the first order in the nonlinear coupling parameter $b$. We observed that the critical temperature $T_{c}$ decreases with increasing the nonlinear parameter $b$, which means that the condensation become harder to be formed for higher nonlinear corrections. This result is similar to the holographic $s$-wave superconductors with BI nonlinear electrodynamics [33]. However, we found that the EN electrodynamics has stronger effect on the condensation formation compared to the BI electrodynamics. The analytical results obtained in this work are in good agreement with the existing numerical results for the holographic superconductors with EN electrodynamics [32]. The critical exponent of the condensation also comes out to be $1 / 2$ which is the universal value in the mean field theory. Thus, the superconductor phase transition appeared in the linear Maxwell electrodynamics also exists in its nonlinear extension. Our work helps to give a better understanding of the holographic superconductors when the gauge field is in the form of EN electrodynamics.

Finally, we would like to mention that in this work we only considered the probe limit. It would be interesting if one could generalize this study away from the probe limit, i.e., by taking the backreaction of scalar and gauge fields on the metric background into account, and analytically discuss the effects of both backreaction and the nonlinear coupling parameters on the properties of the holographic superconductors. It is also worthy to extend this investigation to higher curvature corrections theories such as Gauss-Bonnet gravity. These issues are now under investigation and the results will be appeared in the near future.

\section{Conflict of Interests}

The author declares no conflict of interests for the present paper.

\section{Acknowledgments}

We thank Shiraz University Research Council. This work has been supported financially by Research Institute for Astronomy and Astrophysics of Maragha (RIAAM), Iran.

[1] J. M. Maldacena, Adv. Theor. Math. Phys. vol. 2, 231 (1998).

[2] S. S. Gubser, I. R. Klebanov, and A. M. Polyakov, Phys. Lett. B 428, 105 (1998) [arXiv:hep-th/9802109].

[3] E. Witten, Adv. Theor. Math. Phys. 2, 253 (1998) [arXiv:hep-th/9802150].

[4] S. A. Hartnoll, C. P. Herzog and G. T. Horowitz, Phys. Rev. Lett. 101, 031601 (2008) [arXiv:0803.3295 [hep-th]].

[5] S. A. Hartnoll, C. P. Herzog and G. T. Horowitz, JHEP 12, 015 (2008) [arXiv:0810.1563].

[6] O. C. Umeh, JHEP 08, 062 (2009) [arXiv:0907.3136].

[7] I. Amado, M. Kaminski, and K. Landsteiner, JHEP 05, 021 (2009) [arXiv:0903.2209]. 
[8] J. Sonner, Phys. Rev. D 80, 084031 (2009) [arXiv:0903.0627].

[9] S. Franco, A. M. Garcia-Garcia, and D. Rodriguez-Gomez, Phys. Rev. D 81, 041901 (2010)[arXiv:0911.1354].

[10] C. P. Herzog, Phys. Rev. D 81, 126009 (2010) [arXiv:1003.3278[hep-th]].

[11] G. T. Horowitz and M. M. Roberts, Phys. Rev. D 78, 126008 (2008) [arXiv:0810.1077].

[12] G. Siopsis and J. Therrien, JHEP 05, 013 (2010) [arXiv:1003.4275].

[13] K. Maeda, M. Natsuume, and T. Okamura, Phys. Rev. D 79, 126004 (2009) [arXiv:0904.1914].

[14] Q. Y. pan and B. Wang, Phys. Lett. B 693, 159 (2010) [arXiv:1005.4743].

[15] X. H. Ge, B. Wang, S. F. Wu and G. H. Yang, JHEP 08, 108 (2010) [arXiv:1002.4901].

[16] R. G. Cai, Z.Y. Nie, H.Q. Zhang, Phys. Rev. D 82, 066007 (2010) [arXiv:1007.3321]; Rong-Gen Cai, Huai-Fan Li, and H.Q. Zhang, Phys. Rev. D 83, 126007 (2011) [arXiv:1103.5568]; R. G. Cai, L. Li, L. F. Li, JHEP 1401, 032 (2014) [arXiv:1309.4877].

[17] X.M. Kuang, E. Papantonopoulos, G. Siopsis, B. Wang, Phys. Rev. D 88, 086008 (2013), [ arXiv:1303.2575].

[18] J.P. Wu, Y. Cao, X.M. Kuang, W.J. Li, Phys. Lett. B 697,153 (2011).

[19] H.B. Zeng, X. Gao, Y. Jiang, H.S. Zong, JHEP, 05, 002 (2011).

[20] G. Siopsis and J. Therrien, JHEP 05, 013 (2010) [arXiv:1003.4275].

[21] S. Gangopadhyay and D. Roychowdhury, JHEP 08, 104 (2012) [arXiv:1207.5605 [hep-th]].

[22] R. Banerjee, S. Gangopadhyay, D. Roychowdhury and, A. Lala, Phys. Rev. D 87, 104001 (2013).

[23] C. M. Chen, M. F. Wu, Prog. Theor. Phys. 126, 387 (2011).

[24] J. Jing, Q. Pan, S. Chen, JHEP 11, 045 (2011) [arXiv:1106.5181].

[25] X. H. Ge, S. F. Tu, B. Wang, JHEP 09, 088 (2012) [arXiv:1209.4272];

X. H. Ge and H. Q. Leng, Prog. Theor. Phys. 128, 1211 (2012)[arXiv:1105.4333].

[26] W. Yao, J. Jing, JHEP 05, 101 (2013) [arXiv:1306.0064]; J. Jing, L. Wang, Q. Pan, S. Chen, Phys. Rev. D 83, 066010 (2011).

[27] C. Lai, Q. Pan, J. Jing, and Y. Wang, Phys. Lett. B 749, 437 (2015), [arXiv:1508.05926].

[28] Y. Liu, Y. Gong, and B. Wang, JHEP 02 (2016) 116, [arXiv:1505.03603].

[29] S. H. Hendi, JHEP 03, 065 (2012);

S. H. Hendi, A. Sheykhi, Phys. Rev D 88, 044044 (2013) [arXiv:1405.6998].

[30] A. Sheykhi, S. Hajkhalili, Phys. Rev. D 89 (2014) 104019;

A. Sheykhi, A. Kazemi, Phys. Rev. D 90 (2014) 044028;

A. Sheykhi and S.H. Hendi, Can. J. Phys. 94 (2016) 58;

A. Sheykhi, Adv. in High Energy Phys., Vol. 2014, Article ID 615041.

[31] J. Jing and S. Chen, Phys. Lett. B 686 (2010) 68 [arXiv:1001.4227].

[32] Z. Zhao, Q. Pan, S. Chen, and J. Jing, Nucl. Phys. B 871, 98 (2013) [arXiv:1212.6693].

[33] S. Gangopadhyay and D. Roychowdhury, JHEP 05, 002 (2012) [arXiv:1201.6520].

[34] J. Jing, Q. Pan and S. Chen, JHEP 11, 045 (2011) [arXiv:1106.5181];

J. Jing, L. Jiang and Q. Pan, Class. Quantum Grav. 33, 025001 (2016);

A. Sheykhi, H. R. Salahi and A. Montakhab, JHEP 04, 058 (2016), [ arXiv:1603.00075];

H. R. Salahi, A. Sheykhi, A. Montakhab, [ arXiv:1608.05025];

A. Sheykhi, F. Shamsi, [ arXiv:1603.02678].

[35] S. Gangopadhyay and D. Roychowdhury, JHEP 05, 156 (2012) [arXiv:1204.0673].

[36] A. Sheykhi, F. Shaker, Phys. Lett. B 754, 281 (2016). [arXiv:1601.04035].

[37] D. Ghoraia, S. Gangopadhyay, Eur.Phys. J. C, DOI:10.1140/epjc/s10052-016-4005-0, [arXiv:1511.02444].

[38] P. Breitenloher and D. Z. Freedman, Ann. Phys. (N.Y.) 144, 249 (1982).

[39] M. Abramowitz and I. A. Stegun, Handbook of Mathematical Functions, Dover, New York, (1972). 